\title{
Modelamiento geoestadístico de mediciones de concentración de material particulado (PM10) para la validación de un método simplificado
}

\author{
Geostatistical modeling of particulate matter (PM10) concentration measurements for validation of \\ a simplified method \\ Lenin Javier Ramírez-Cando,2*, Miguel Armijos ${ }^{1}$, Michelle Crespo ${ }^{1}$, Sandra Paulina Pino-Casignia ${ }^{1}$ y César Iván \\ Álvarez-Mendoza ${ }^{1,2}$ \\ * Autor de correspondencia
}

\section{Resumen}

El material particulado (PM10), es un contaminante atmosférico capaz de ocasionar una grave problemática en cuanto a calidad del aire y afecciones en la salud humana. Por lo que en la presente investigación se presenta el análisis de las concentraciones de material particulado (PM10) en el sector del aeropuerto de Tababela en el Distrito Metropolitano de Quito, cuyo objetivo es determinar el método más apropiado para la medición de concentración de material particulado (PM10), mediante análisis geoestadístico de tres métodos: IDW, Spline y Kriging por medio del Software ArcGIS, con la finalidad de identificar los puntos de mayor concentración de PM10 en la zona de estudio. La toma de datos se realizó utilizando un muestreador casero, al igual que los datos obtenidos de la Red Metropolitana de Monitoreo Atmosférico de Quito (REMMAQ) ubicada en el sector de Carapungo; con estos se valida la metodología de muestreo mediante el método de regresión lineal o mínimos cuadrados, se muestra la utilidad del muestreador casero. Se recolectó los datos de concentración en 16 puntos estratégicos con duplicados cada uno que cubren la ruta Collas, con los que se obtuvo mapas de la zona analizada uno por cada método propuesto, los mismos se validaron mediante la aplicación de la herramienta Arctoolbox relacionando los datos obtenidos con respecto al blanco, se presenta que el valor de incertidumbre indica una proximidad en relación a la concentración real; siendo el modelo Inverse distance weighted (IDW) el que respondió de mejor manera al comportamiento de PM10 en la zona permitiendo así obtener el mejor modelo que diferencie los puntos de menor y mayor concentración de PM10, a diferencia de los modelos Spline y Kriging.

Palabras clave: material particulado; geoestadístico; Inverse distance weighted; kriging; spline; validación; calidad del aire.

\begin{abstract}
The particulate material (PM10), is one atmospheric pollutant capable of causing a serious problem in terms of air quality and human health conditions. For this reason, the present investigation presents the analysis of particulate material concentrations (PM10) in the Tababela airport sector in the Metropolitan District of Quito, whose objective is to determine the most appropriate method for the measurement of particulate matter concentration. (PM10), by geostatistical analysis of three methods: IDW, Spline and Kriging by means of the ArcGIS Software, in order to identify the points with the highest concentration of PM10 in the study area. The data collection was done using a home sampler, as well as the data obtained from the Metropolitan Atmospheric Monitoring Network of Quito located in the Carapungo sector; with these the sampling methodology is validated by the linear regression or least squares method, the utility of the home sampler is shown. The concentration data was collected in 16 strategic points with duplicates each covering the Collas route, with which maps of the analyzed area were obtained one for each proposed method, they were validated by applying the Arctoolbox tool relating the data obtained with respect to the target, it is presented that the uncertainty value indicates a proximity in relation to the real concentration; The Inverse Distance Weighted (IDW) model was the one that responded better to the behavior of PM10 in the area, thus allowing us to obtain the best model that differentiates the points of lower and higher concentration of PM10, unlike the Spline and Kriging models.
\end{abstract}

Keywords: particulate matter; geostatistics; Inverse distance weighted; kriging; spline; validation; air quality.

\section{Introducción}

El material particulado se define como el conjunto de materia dispersada en la atmósfera y condensada en forma sólida o líquida emitidas directamente al aire, tales como el hollín de diésel, polvo de vías y las partículas resultantes de procesos productivos (Arciniégas, 2012), 
es denominado como un contaminante primario generado por la combustión ineficiente de combustibles fósiles, el mayor precursor es la combustión de diesel (Gaviria et al., 2011). El material particulado está formado por una mezcla compleja de componentes cuya composición incluye compuestos orgánicos, sulfatos, nitratos u óxidos de silicio (Seinfeld, 2006).

Dependiendo de su fuente de origen, se componen de una mezcla de sustancias orgánicas e inorgánicas que presentan una composición física y química variable (Vivar, 2014). El material particulado 2,5 o PM 2.5 es aquel que tiene un diámetro aerodinámico menor a 2,5 micrómetros y PM10 a aquel cuyo diámetro aerodinámico es menor a 10 micrómetros (Diaz, 2015). El material particulado sedimentable, es el material particulado, sólido o líquido, cuyo tamaño es mayor a 10 micrómetros y que es capaz de permanecer en suspensión temporal en el aire ambiente (Maldonado, 2012).

El desarrollo del estudio se da en base a la influencia que tiene el Aeropuerto de Tababela como foco de emisión de material particulado PM10 debido al despegue y aterrizaje de aviones (Urbaneja, 2016)

El departamento Social Europeo y la Aviación Danesa, nos indica, que los aeropuertos son considerados como fuentes de contaminación del aire, debido a los gases de escape, emitidos por los aviones y los motores diésel, las emisiones directas de combustible durante el reabastecimiento de los aviones y las partículas de mayor tamaño generadas por los frenos, los neumáticos, el asfalto, el suelo, etc. Como emisores de altas concentraciones de material particulado (PM10) (The ecological council, 2012).

Las pruebas relativas al material particulado suspendido en el aire y sus efectos en la salud pública coinciden en poner de manifiesto efectos adversos para la salud con las exposiciones que experimentan actualmente las poblaciones urbanas, tanto en los países desarrollados como en los que se encuentran en vías de desarrollo, y estas afecciones causan un daño mayor al sistema respiratorio. (Organización Mundial de la Salud [OMS], 2005)

Por esta razón se han establecido umbrales y límites permisibles en cuanto a las normativas que rigen en cada país, para evitar consecuencias graves en cuanto a problemas de salud pública (Vivar, 2014). Los factores más importantes para la absorción en el sistema respiratorio de los contaminantes presentes en partículas son su tamaño y solubilidad en agua (Ruiz, 2006), las partículas de tamaño inferior a $10 \mu \mathrm{m}$ penetran en los pulmones. Las de tamaño comprendido en el intervalo $2,5-10 \mu \mathrm{m}$ se depositan en la tráquea, bronquios y bronquiolos (Gaviria et al., 2011). El sistema respiratorio puede ser dañado directamente por material particulado que entra al sistema sanguíneo o linfático a través de los pulmones. Además, los componentes solubles del material particulado pueden ser transportados a órganos distantes y causar efectos nocivos sobre estos. (Golato et al,. 2012).
El material particulado es considerado como uno de los principales problemas de contaminación del Distrito Metropolitano de Quito. Los límites máximos permitidos establecidos en la legislación ecuatoriana son $50 \mu \mathrm{g} / \mathrm{m}^{3}$ como promedio anual y $100 \mu \mathrm{g} / \mathrm{m}^{3}$ como promedio en 24 horas para PM 10 (Maldonado, 2012).

En ArcGIS, se ingresa información sobre los atributos la cual sirve para crear mapas, los cuales se pueden analizar de manera visual (ESRI, 2016). Así, el análisis geoestadístico ayuda a extraer los datos que no se pueden apreciar a simple vista en un GIS, revelando características de un conjunto de entidades como uno solo, se utiliza para analizar la distribución que tiene un atributo en particular, para encontrar valores que sean extremadamente bajos o altos (ESRI, 2016).

La interpolación puede utilizarse para predecir valores no conocidos de cualquier dato en un punto geográfico a partir de una cantidad limitada de datos obtenidos para una muestra (ESRI, 2016). Los métodos de interpolación disponibles para ArcGIS son: ponderación de distancia inversa (IDW), el cual interpola una superficie de un ráster a partir de los valores de los puntos muestreados, calcula promedios de estos valores utilizando como técnica la distancia inversa ponderada (ESRI, 2016).

Spline estima valores utilizando una función matemática que minimiza la curvatura de la superficie generada con los datos muestreados, la cual pasa por los puntos de entrada (ESRI, 2016). Kriging, a partir de un conjunto de datos genera una superficie, los datos que utiliza se encuentran dispersados con valores distintos de z, razón por la cual este método requiere una investigación más exhaustiva antes de seleccionar el método de estimación más conveniente (ESRI, 2016).

El objetivo principal de este artículo, es determinar el método más apropiado para la medición de concentración de material particulado (PM10), mediante análisis geoestadístico de tres métodos (IDW, SPLINE, KRIGING) por medio del Software ArcGIS, con la finalidad de identificar los puntos de mayor concentración de PM10 en la zona de estudio.

\section{Materiales y metodología}

El inicio del ensayo se realizó de 8:00 a 16:00, debido a la iluminación. En un lugar donde la sombra de los alrededores no se proyecte sobre la superficie del filtro muestreador. El método es más eficiente, cuando no se registran vientos fuertes, ni precipitaciones, durante el muestreo, de tal manera que la superficie del aparato no se vea alterada. La medición se realizó cada 24 horas debido a la dificultad de movilización a la zona de muestreo.

\section{Proceso de fabricación del muestreado}

Colocar el papel filtro alrededor del tubo PVC y fijarlo con una banda elástica. Luego, realizar una especie de caja hueca de cuatro caras y proceder a forrar la caja con papel 
contact para impermeabilizarla. La caja que será colocada sobre el tubo PVC permitirá que actué como cabezal de corte para absorber las partículas de PM10, ya que el aire ingresa a través de la abertura ubicada en la parte lateral del cabezal de corte para PM10, que fue ubicado de manera horizontal tomando como referencia el suelo, dependiendo de la dirección del viento que será lo que permite aspirar el material particulado durante 24 horas. Y, proceder a realizar orificios en la mitad del tubo de PVC y fijar esta estructura a la caja de cartón, para posteriormente poder atravesarla con el soporte de 1,5 m. Fijar correctamente la estructura enterrándola en la tierra, dejando el filtro a una altura de $1 \mathrm{~m}$ exponiéndolo en dirección del viento proveniente de la zona con posible carga contaminante.

\section{Proceso de toma de muestras en campo}

Se realizó un mapa con coordenadas y puntos de muestreo en la zona de estudio usando ArcGIS y Google Earth (Figura 1y 2).

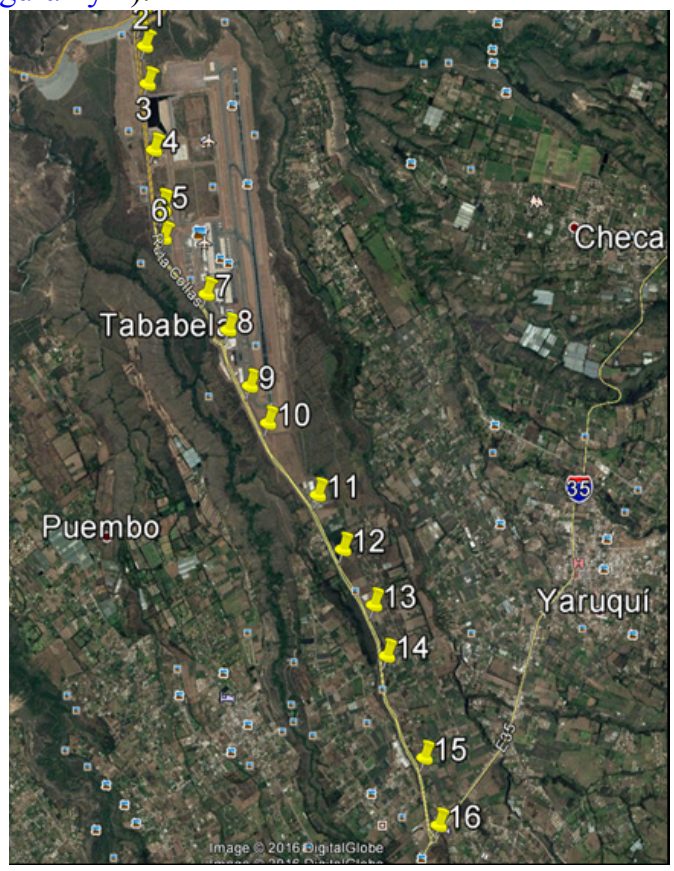

Figura 1. Puntos de muestreo Software Google earth

Se colocó el medidor casero a una altura mínima de $1 \mathrm{~m}$ respecto de la superficie del suelo, verificando la horizontalidad con un nivel. Se fijó la base, de manera que esté estable y no se vea afectado por la fuerza del viento, el cual podría ocasionar que se voltee. Se acompañó a cada lote de filtros muestreados con un filtro blanco, el cual fue sometido a las mismas manipulaciones, excepto que no fue expuesto a la contaminación de la zona. Se utilizaron filtros de fibra de cuarzo de 8 " * 10", de poro 2 um, el cual una vez dentro del cabezal de muestreo, el flujo de aire pasa a través del muestreador y son dirigidos a la superficie de impactación, las partículas con tamaños mayores a 10um quedan retenidas en el filtro mientras que el aire limpio es expulsado por el extremo opuesto. Se dejó el medidor casero durante 24 horas. Después de dicho período se procedió a recolectar las muestras en diferentes fundas ziploc y se la almacenó en conjunto en una caja para poder transportar las muestra de manera cuidadosa, evitando cualquier tipo de daño o perdida de material que pueda afectar el momento de su análisis. Para luego, codificar cada filtro para conocer el punto que representa en la zona de muestreo.

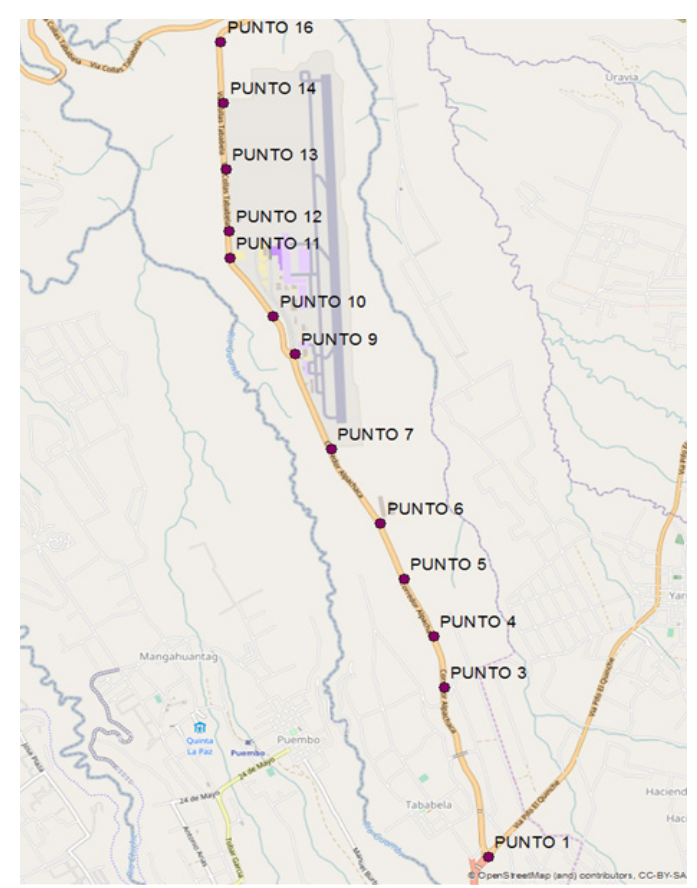

Figura 2. Puntos de muestreo Software ArcGis versión 10.0

\section{Procedimiento de análisis gravimétrico}

La limpieza del material para la medición en laboratorio de pesos de la muestra debe ser adecuada, para lo cual se debe limpiar con una brocha pequeña el área próxima a las balanzas debe mantenerse libre de polvo. Las pinzas deben limpiarse cuidadosamente usando alcohol etílico. Luego, extraer los filtros de muestreo cuidadosamente de la caja de almacenamiento y de la bolsa Ziploc.Previo a la medición de pesos se debe calibran la balanza con la metodología de calibración del equipo, se ajusta el cero de la balanza.

Se determina el peso de los filtros, obteniendo el peso de cada uno, sin exponerlos al medio de la zona de muestreo. Una vez determinado el peso de cada filtro con el material particulado se anota el dato con la codificación de la muestra y el respectivo peso en una ficha.

Para obtener el peso de PM10 se realiza una diferencia entre el peso del filtro con la muestra menos la media del filtro limpio. La concentración del polvo o material particulado en el aire muestreado, se obtiene remplazando datos en la siguiente fórmula.

$$
\text { Concentración } n=\frac{\text { Peso Material particulado } \quad[\mathrm{mg}]}{\text { Volumen de aire muemstreado }[\mathrm{m} 3]}
$$


El dato de volumen de aire se obtiene con respecto al volumen del cilindro usando la siguiente formula.

$$
V=\pi \cdot r^{2} \cdot h
$$

Siendo $\mathrm{r}$ el radios del tubo de PVC y h la altura del mismo. (Figura 3).

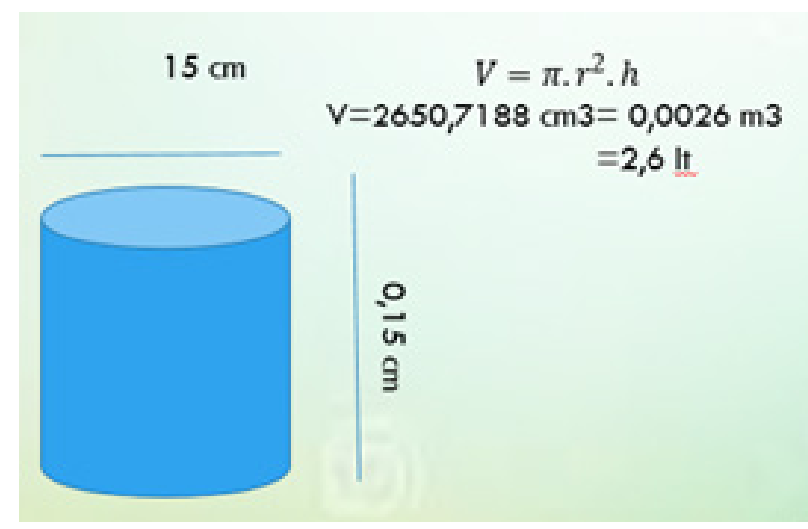

Figura 3. Cálculo de volumen del tubo de pvc

En excel se realizará el tratamiento de todos los datos de peso y volumen con fórmulas programadas para facilitar el proceso de obtención de concentración por cada punto muestreado .(Tabla 1)

\section{Validación del metodo de muestreo}

Se aplicó la regresión lineal o mínimos cuadrados debido a que se utiliza tanto para pronósticos de series de tiempo, como para pronósticos de relaciones causales.

Las variables candidatas a ser consideradas para generar la ecuación de regresión son de 36 datos de muestra de $\mathrm{PM} 10 \mathrm{ug} / \mathrm{m}^{3}$.

El uso del Software de Matlab, se aplicó de forma fácil, esta regresión, siguiendo los siguientes pasos simplificados:

- El usuario debe checar primero las instrucciones del programa para entender el funcionamiento del programa y no tenga ningún problema al usarlo.

- El usuario debe de proporcionar los puntos de x (Son datos de la estación de Carapungo de la REMMAQ que usa datos certeros mediante estaciones digitales).

- El usuario deberá introducir los valores de los puntos de y (Son los datos obtenidos con 5 duplicados durante seis días).

- Una vez introducidos los datos de concentración en la programación con el algoritmo aplicado, se obtendrá la ecuación lineal dependiente de estos puntos proporcionados generando una gráfica que se trata de la siguiente forma:

- Ingresar en la barra de herramientas de la gráfica en Tools

- Ir a la opción Basic Fitting
- Seleccionar la opción de lineal con splain interpolant, el cual denotara el valor del grado de confiabilidad del método. Valor que debe de ser cercano a 1 para la aceptación del método.

\section{Validación del modelo geoestadístico}

Según García (2002), en las mediciones de partículas hay muchas causas de error, estas incluyen interferencias, niveles de blancos, reproducibilidad de los métodos de muestreo y muestreo estadístico. Por lo cual se lleva a cabo una suma de acumulados de las concentraciones de muestreo, respecto a todos los días para cada duplicado

Los datos obtenidos de los duplicados acumulados se separan en un nuevo libro de Excel para poder ser vinculados al Software ArcGIS versión 10.4.1.

Se procede a georreferenciar las coordenadas de los puntos de muestreo para poder enlazar el mapa de la zona analizada.

Para la validación de los tres modelos de concentración se aplica la herramienta Arctoolbox en la cual se encontrará las opciones de modelación (IDW, SPLINE Y KRIGING). Relacionando datos obtenidos en cada método con respecto al blanco analizado.

Para IDW se conecta la carpeta en la que tenemos el archivo de Excel con los datos muestreados, en el atributo de $\mathrm{z}$ value field colocamos la opción de las concentraciones muestreadas, se selecciona la carpeta en la que se guardara el ráster de salida, se selecciona $\mathrm{OK}$ y se genera una superficie la cual al ser interpolada debe ser el de una variable que sea dependiente de la ubicación de la zona muestreada, ya que se puede limitar los puntos previos a la interpolación y el método supone que la variable tomada disminuye la influencia a mayor distancia de la ubicación del punto de muestreo.

Al seleccionar Spline que es un método de interpolación el cual estima valores a través de una función matemática, se conecta el archivo Excel con los datos muestreados, en el atributo de $\mathrm{z}$ value field colocamos la opción de las concentraciones muestreadas, se selecciona la carpeta en la que se guardara el ráster de salida, se selecciona $\mathrm{OK}$ y se genera el mapa; este método es el mejor para generar superficies que varían en cantidades mínimas como las concentraciones de contaminación. A mayor número de puntos más tardará el programa en procesar el ráster de salida.

Kriging es un método de interpolación geoestadístico que genera una superficie a partir de un conjunto de puntos que están dispersados, se conecta la carpeta en la que tenemos el archivo de Excel con los datos muestreados, en el atributo de $\mathrm{z}$ value field colocamos la opción de las concentraciones muestreadas, se selecciona la carpeta en la que se guardara el ráster de salida en output Surface raster seleccionando el método ordinario y en la opción semivariogram model la opción spherical, se selecciona OK tomando como referencia. 
Tabla 1. Concentraciones PM10 en puntos de muestreo

\begin{tabular}{|c|c|c|c|c|c|c|}
\hline & Días & Punto & Muestra 1 & Duplicado 1 & Duplicado 2 & Duplicado 3 \\
\hline 1 & $8 / 1 / 2017$ & Punto 1 & 108,605917 & 92,578877 & 111,291923 & 111,920726 \\
\hline 2 & & Punto 2 & 294,134375 & 273,025625 & 255,435 & 241,305 \\
\hline 3 & & Punto 3 & 290,645 & 275,642656 & 279,611328 & 276,318477 \\
\hline 4 & & Punto 4 & 401,34 & 158,36 & 446,55 & 335,416667 \\
\hline 5 & & Punto 5 & 368,378333 & 246,888333 & 390,983333 & 379,680833 \\
\hline 6 & 9/1/2017 & Punto 1 & 201,370146 & 182,802251 & 183,363461 & 176,612863 \\
\hline 7 & & Punto 2 & 288,195742 & 269,502708 & 283,58 & 283,034619 \\
\hline 8 & & Punto 3 & 260,458164 & 281,595664 & 280,772451 & 274,755078 \\
\hline 9 & & Punto 4 & 313,284583 & 346,482708 & 324,350625 & 305,907222 \\
\hline 10 & & Punto 5 & 283,932778 & 346,403889 & 324,40974 & 329,913203 \\
\hline 11 & $10 / 1 / 2017$ & Punto 1 & 154,988031 & 137,690564 & 147,327692 & 144,266794 \\
\hline 12 & & Punto 2 & 405,233333 & 428,216667 & 275,501667 & 511,981667 \\
\hline 13 & & Punto 3 & 470,099167 & 372,800417 & 421,449792 & 389,016875 \\
\hline 14 & & Punto 4 & 327,131914 & 340,032444 & 320,060301 & 335,416667 \\
\hline 15 & & Punto 5 & 515,4 & 179,51 & 564,66 & 419,856667 \\
\hline 16 & $11 / 1 / 2017$ & Punto 1 & 203,220708 & 133,061734 & 212,07173 & 207,944557 \\
\hline 17 & & Punto 2 & 361,989444 & 346,667222 & 412,894444 & 395,433385 \\
\hline 18 & & Punto 3 & 400,333359 & 394,675117 & 412,5249 & 384,136366 \\
\hline 19 & & Punto 4 & 333,427874 & 306,92299 & 332,664935 & 331,969613 \\
\hline 20 & & Punto 5 & 467,628333 & 299,683333 & 492,258333 & 479,943333 \\
\hline 21 & $12 / 1 / 2017$ & Punto 1 & 286,204775 & 284,999876 & 293,495941 & 288,461959 \\
\hline 22 & & Punto 2 & 405,233333 & 405,659678 & 373,500291 & 402,783346 \\
\hline 23 & & Punto 3 & 318,308633 & 316,432135 & 316,244117 & 300,065481 \\
\hline 24 & & Punto 4 & 324,106826 & 323,788391 & 327,546146 & 333,69314 \\
\hline 25 & & Punto 5 & 435,780556 & 404,158333 & 412,0075 & 408,42125 \\
\hline 26 & $13 / 1 / 2017$ & Punto 6 & 415,768402 & 397,693147 & 409,764318 & 410,768578 \\
\hline 27 & & Punto 7 & 798,053333 & 787,656922 & 709,988364 & 788,559753 \\
\hline 28 & & Punto 8 & 798,053333 & 922,011667 & 574,541667 & 897,606667 \\
\hline 29 & & Punto 9 & 1568,23667 & 1436,49833 & 1150,54333 & 2117,66833 \\
\hline 30 & & Punto 10 & 389,813333 & 434,878333 & 404,835 & 379,798889 \\
\hline 31 & $14 / 1 / 2017$ & Punto 6 & 536,612534 & 572,42074 & 546,083826 & 576,793456 \\
\hline 32 & & Punto 7 & 909,809167 & 742,175417 & 825,992292 & 770,114375 \\
\hline 33 & & Punto 8 & 779,066172 & 774,590273 & 808,858286 & 757,019144 \\
\hline 34 & & Punto 9 & 1777,08333 & 1463,81333 & 1620,44833 & 1516,025 \\
\hline 35 & & Punto 10 & 426,059306 & 398,84287 & 419,856667 & 416,757487 \\
\hline 36 & $15 / 1 / 2017$ & Punto 6 & 873,004494 & 834,855473 & 818,788894 & 854,979001 \\
\hline 37 & & Punto 7 & 487,417826 & 465,132717 & 489,739307 & 490,191345 \\
\hline 38 & & Punto 8 & 846,86758 & 812,703754 & 829,69836 & 807,461896 \\
\hline 39 & & Punto 9 & 1429,00556 & 1516,83111 & 1524,32389 & 1588,11833 \\
\hline
\end{tabular}


Resultando del paso anterior las gráficas ilustrativas respecto a cada modelo en el cual se diferenciara los puntos de menor y mayor concentración de PM10 a lo largo del tramo estudiado.

Para la interpretación del mejor modelo que resulte del análisis se procede a medir el valor de incertidumbre el cual se calcula de la siguiente forma: Esto se puede visualizar en la opción de Identify (i) al generar cada uno de los mapas con las distintas opciones de interpolación, la cual indica valores de proximidad a la concentración real.

\section{Resultados y discusión}

\section{Análisis gravimétrico}

Los resultados obtenidos respecto a pesos, volumen de muestreo, Concentraciones de PM10 en $\mathrm{ug} / \mathrm{m}^{3}$ se observan en la Tabla 1 y los valores acumulados obtenidos en concentración por duplicados se observan en la Tabla 2.

\section{Validación del método de muestreo}

De la Figura 3 se obtiene un grado de confiablidad de 0,9733 que al acercase a 1 nos indica que el método de toma de muestra es eficiente y sus resultados no son distantes a los valores arrojados por la estación digital validada por la REMMAQ.

\section{Validación del modelo geoestadístico}

De los métodos aplicados en el programa ArcGIS para el análisis geoestadístico de material particulado (PM10), utilizando los métodos: IDW (Tabla 2), KRIGING y SPLINE, con los datos muestreados en el tramo de la Ruta Collas - redondel de Tababela, los resultados obtenidos muestran que el método más eficiente en la evaluación de concentración de PM10, es el IDW (Interpolación lineal con la ponderación de la distancia inversa) con un valor de aproximación a la concentración real de 275,1948 $\mathrm{ug} / \mathrm{m}^{3}$, ya que la variable que utiliza es dependiente de la ubicación facilitando la estimación de la misma. En comparación del método SPLINE que destaca valores más lejanos al IDW de 640,6793 $\mathrm{ug} / \mathrm{m}^{3}$. El modelo KRIGING es el menos adecuado para la medición de PM10 debido a que la aproximación a la concentración real de 1204,8642 $\mathrm{ug} / \mathrm{m}^{3}$ y al visualizar el mapa de este modelo genera una percepción menor en cuanto a la dispersión de PM10 a comparación de los dos modelos anteriores a este. (Figura 5 y 6 en cuanto a concentraciones de PM 10 con el modelo SPLINE Y KRIGING)

En la Figura 4, se ilustran las gráficas de los modelos aplicados a los puntos de muestreo, respecto a la concentración de PM10.

A través del método IDW se pudo obtener una representación cartográfica de la concentración de PM10,
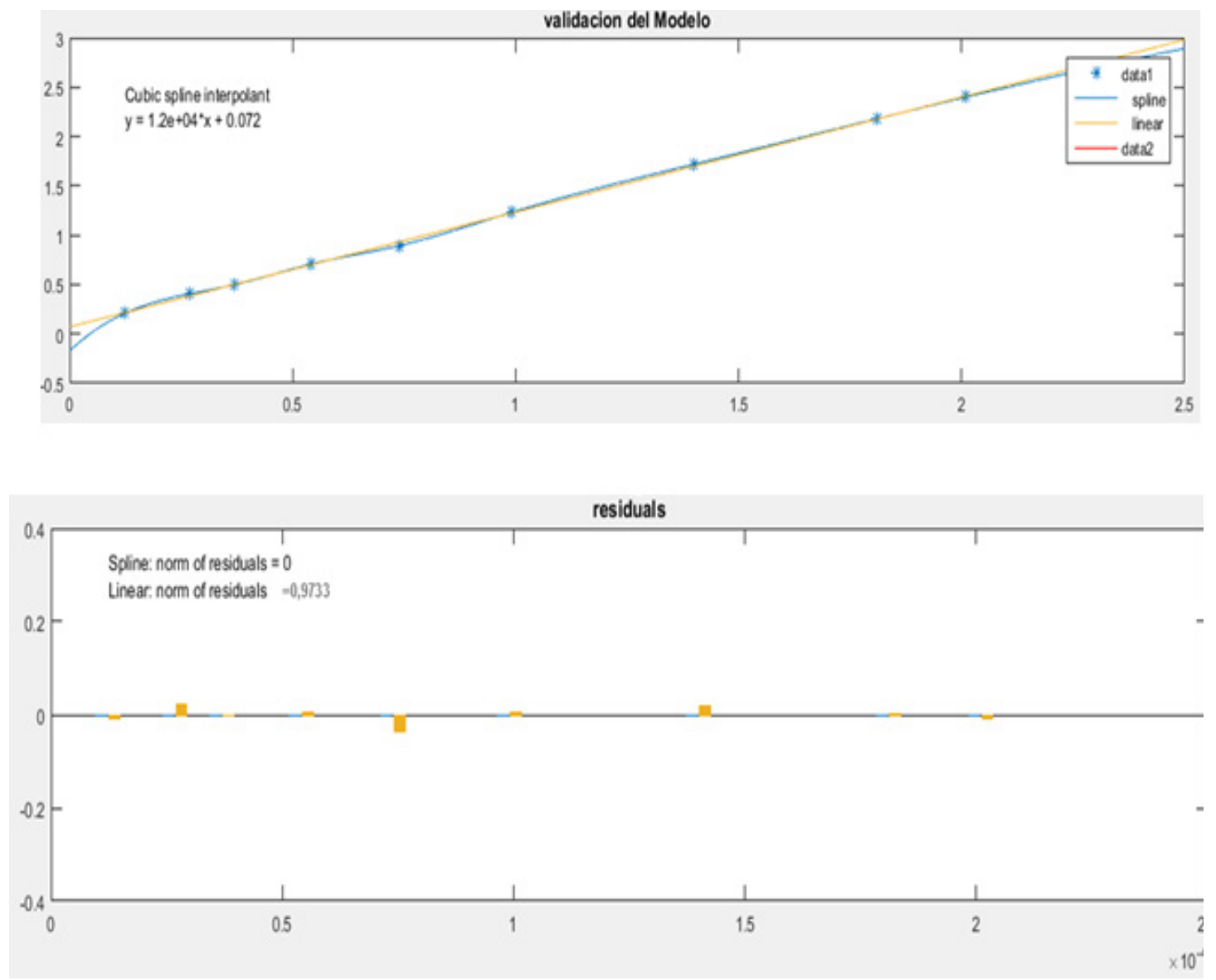

Figura 3. Gráfica de regresión lineal (análisis de residuos) 
en el que se observa que los puntos que presentan mayor concentración de material particulado, superando el límite máximo permisible de $100 \mathrm{ug} / \mathrm{m}^{3}$ valor establecido en la normativa ecuatoriana vigente y que respecto a el índice de calidad del aire se considera como sitios emergentes a los puntos 8,9,10,11,14 y 16, sin embargo los puntos más críticos en comparación a los otros es el punto 9, ubicado expuestas a PM10 tienen un riesgo de muerte prematura o agravamiento de la enfermedad (Secretaria de Ambiente, 2015). En niños con enfermedades pulmonares se puede apreciar una disminución en la capacidad para respirar de manera profunda, experimentando síntomas de agitación (Secretaria de Ambiente, 2015); en adultos mayores se puede agravar los cuadros de enfermedades respiratorias o

Tabla 2. Tabla comparativa de los resultados de muestreo respecto a blanco con el modelo IDW

\begin{tabular}{cccccc}
\hline & Blanco punto 8 & Punto 7 muestreado & Blanco punto 2 & $\begin{array}{c}\text { Punto 7 } \\
\text { muestreado }\end{array}$ \\
\hline Idw_pixel value & 4508,875488 & 4233,680664 & Idw_pixel value & 4508,875488 & 4233,680664 \\
\hline
\end{tabular}

en la zona de despegue de aviones del aeropuerto, como también en el redondel donde la afluencia de vehículos es muy alta en los puntos 10 y 11 que resultarían de la dispersión del material particulado.

Los resultados obtenidos de concentración de PM10 fueron comparados con el Índice Quiteño de la Calidad del Aire (IQCA), con el cuál se obtuvo un valor resultante de 275,1948 $\mathrm{ug} / \mathrm{m}^{3}$ con el método IDW de interpolación en el programa ArcGIS versión 10.4.1, valor que se ubica en categoría de nivel de alerta, que en condiciones desde el punto de vista de la salud es no saludable para individuos sensibles (enfermos) (IQCA, 2015); ya que puede en personas que presentan enfermedades de pulmones o corazón causar enfermedades a los pulmones o al corazón (Cortés \& Osses, 2004), como: obstrucción pulmonar crónica o congestiones cardiacas si están

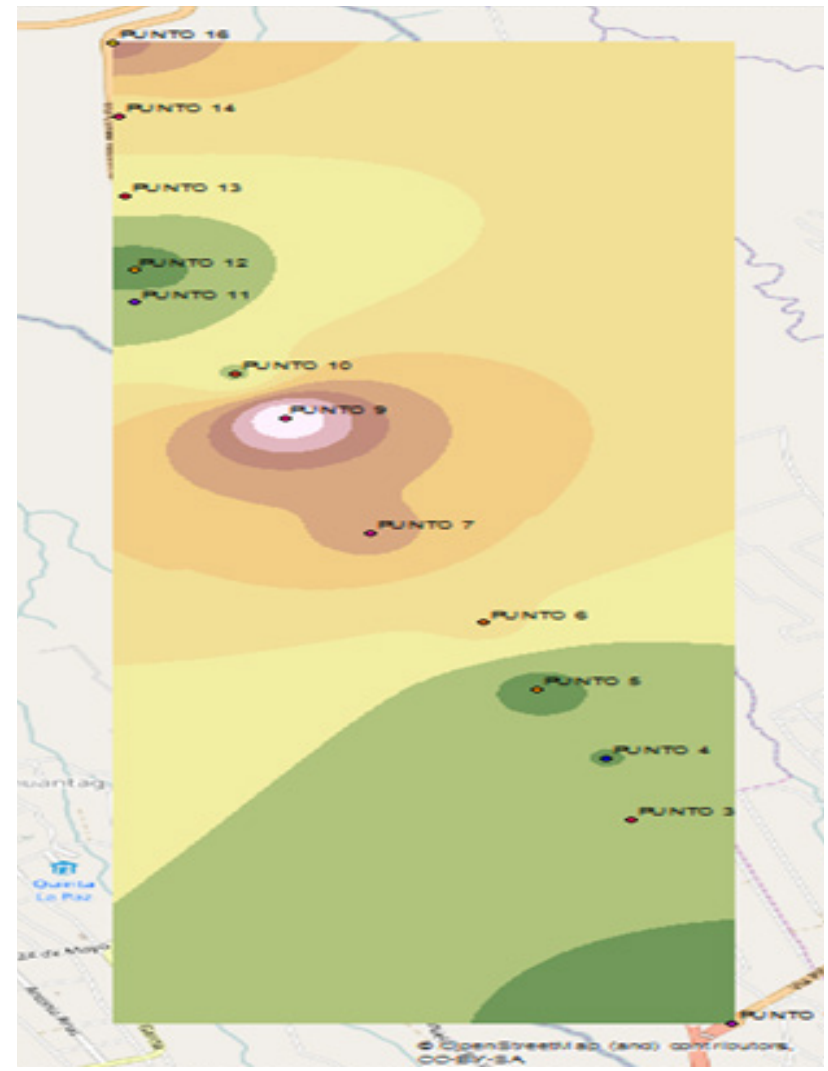

Figura 4. Concentraciones de PM10 modelo IDW

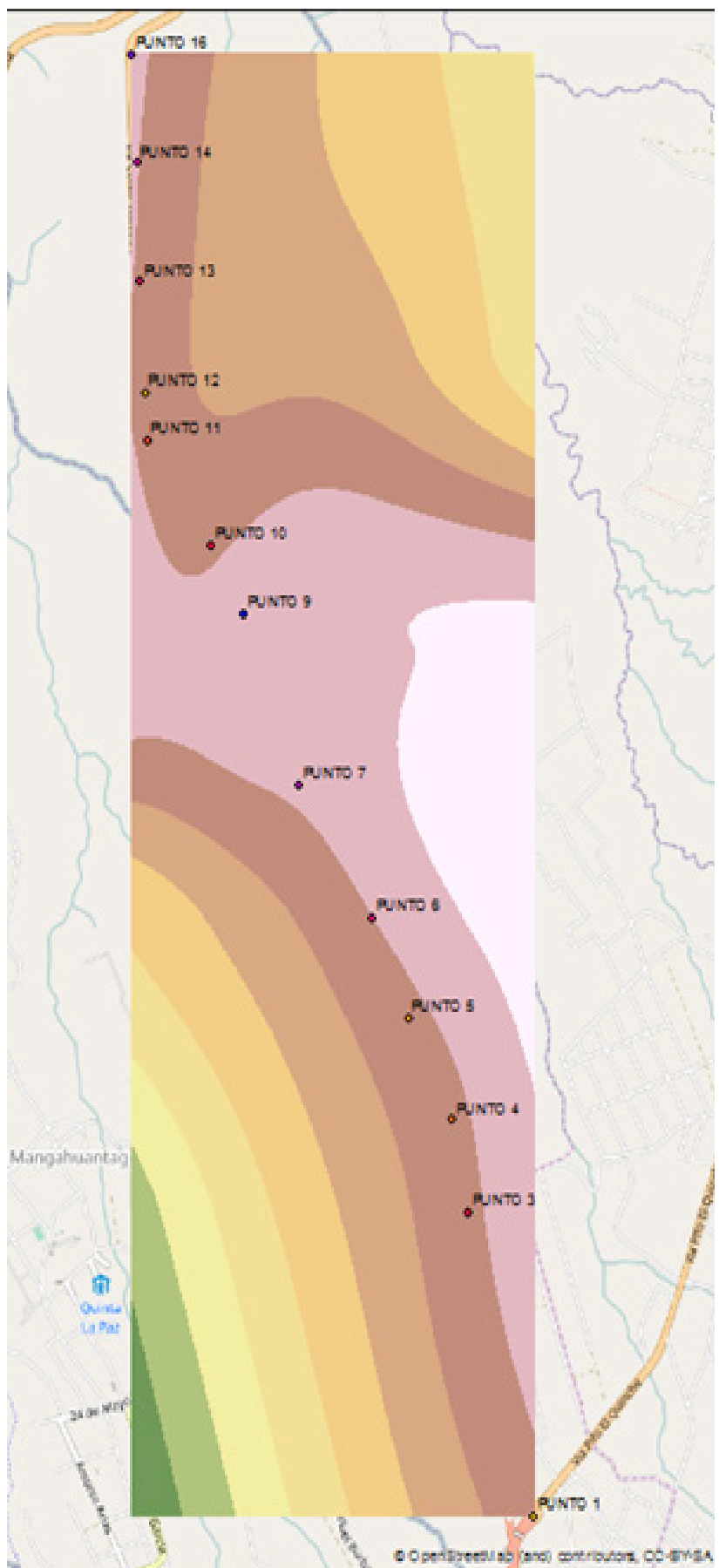

Figura 5. Concentraciones de PM10 modelo SPLINE 


\section{〈INGING DUPLICADO1}

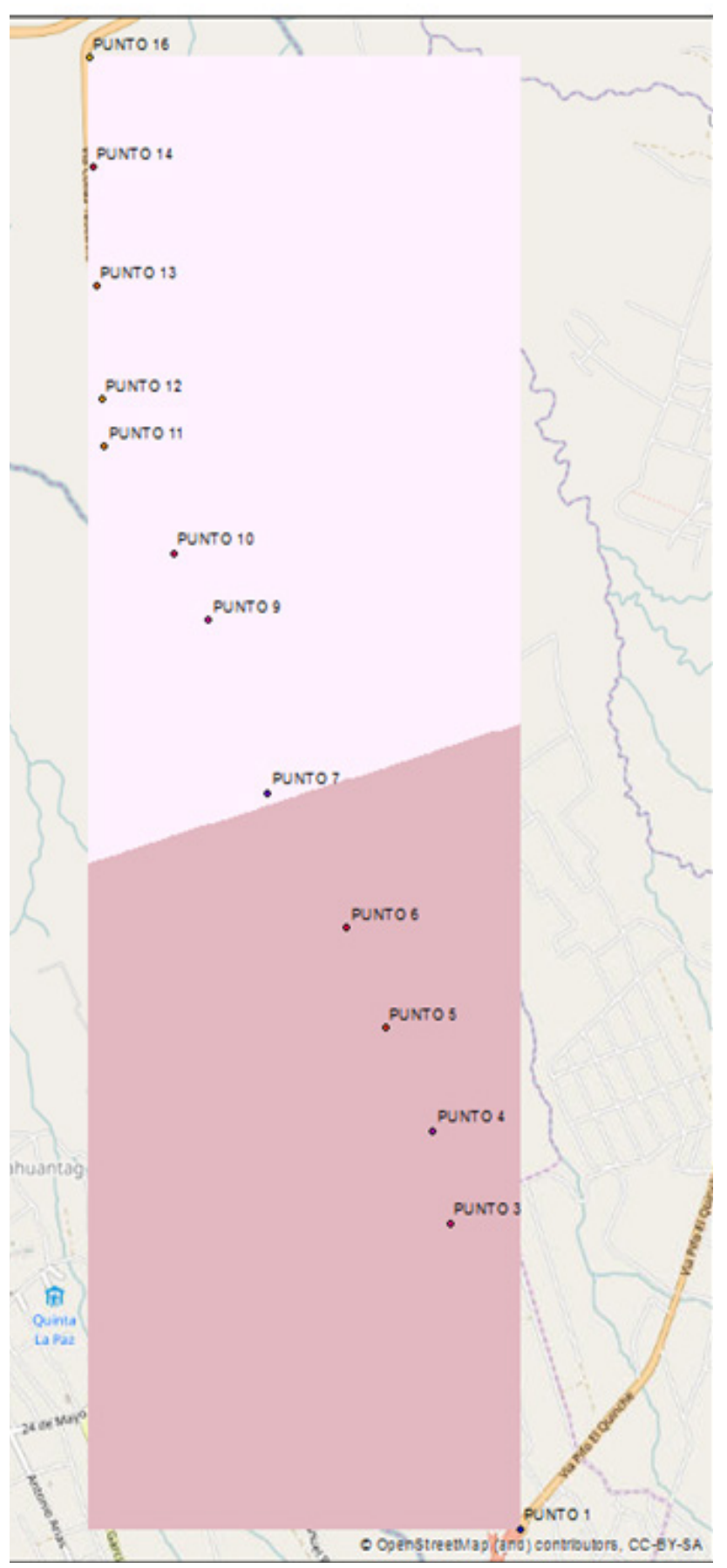

Figura 6. Concentraciones de PM10 modelo RIGING

cardíacas existentes o desarrollar este tipo de problemas en el caso de que no los haya habido (Secretaria de Ambiente, 2015).

La Normativa de Calidad del Aire de Quito en la Tabla 3 (Secretaria de Ambiente, 2015), en cuanto a mediciones de concentraciones de contaminantes de aire establece el método gravimétrico, mediante muestreadores de alto y bajo caudal que permitan separar las partículas por tamaño, mediante el peso ganado por el filtro dividido para el volumen total en un tiempo de muestreo de 24 horas por lo que el muestreador se diseñó y utilizó siguiendo la normativa y sería aplicable para un posible nuevo estudio.

Tomando en cuenta las muestras tomadas para el análisis de las variables seleccionadas concentración Material Particulado (PM10) en la zona de Tababela, misma en la que está ubicado el nuevo aeropuerto de Quito-Ecuador. Para el período de 08 de enero 2017 a 22 de enero del mismo año mismo en el que se realizó el muestreo, tenemos que el método que mejor se comporta de los métodos utilizados es el IDW (Interpolación lineal con la ponderación de la distancia inversa) con un valor de aproximación a la concentración real de 275,1948 ug/ $m^{3}$, que proporciona resultados más satisfactorios, en comparación al método Spline que nos muestra un valor de $640,6793 \mathrm{ug} / \mathrm{m}^{3}$ que está muy alejado al valor que se tiene con el modelo IDW y el modelo KRIGING que se observa seria el menos adecuado ya que tiene una aproximación a la concentración real de 1204,8642 $\mathrm{ug} / \mathrm{m}^{3}$ y al visualizar los mapas resultantes (Figura 4, 5 y 6) de los tres diferentes modelos se observa también una mejor percepción en el mapa IDW en comparación a Spline y Kriging, siendo el que menos percepción proporciona el de Kriging. En el año 2010, se realizó algunos estudios, en cuyos mapas resultantes utilizando el programa ArcGIS versión 10.4.1 con los métodos de interpolación IDW y kriging, el mapa modelado por IDW muestra una mejor percepción en cuanto a contaminación en comparación al mapa modelado por Kriging, teniendo los dos una misma distribución espacial, esto se puede dar porque el modelado realizado con kriging elimina los valores más altos y también los más bajos, lo que produce un suavizado generalizado en el modelo, lo que no ocurre con IDW el cual tiende a crear puntos de concentración en torno a los puntos muestreados. Lo mismo menciona (Canales-Rodríguez et al.,2014) al observar sus mapas resultantes en cuanto a distribución espacial de PM10, obteniendo un mapa modelado con IDW y otro con kriging, en los cuales el mejor método utilizado fue el de IDW en el que se pudo visualizar en el mapa de IDW estimaciones que no obtuvieron en el mapa modelado con kriging, ya que el método kriging logra minimizar el error de predicción reflejando un suavizado en la estimación de los valores.

Tomando los resultados del modelo IDW que fue el modelo más idóneo en el estudio, se pudo obtener una representación cartográfica de la concentración de PM10, estimando los puntos de concentración mayores que fueron los que sobrepasan el límite máximo permisible de $100 \mathrm{ug} /$ $m^{3}$ valor establecido en la normativa ecuatoriana vigente, siendo el punto 9 un punto considerado emergente en comparación a los otros que a pesar de tener concentraciones que sobrepasan el límite máximo permisible no se considera como emergentes lo que representa una seria problemática en el sector y por tanto podría ocasionar afecciones muy serias a la salud y al ambiente. Analizando el comportamiento en cuanto a concentraciones de PM10 se puede contribuir a establecer estrategias y tomar decisiones respecto a los problemas ambientales. 
Tabla 3. Valores acumulados de concentración de PM 10 por duplicado

\begin{tabular}{|l|r|r|r|}
\hline \multicolumn{5}{|c}{ DUPLICADO 1 } \\
\hline PUNTO 1 & $-0,190438$ & $-78,338554$ & 831,1333013 \\
\hline PUNTO 3 & $-0,171984$ & $-78,343045$ & 1641,14599 \\
\hline PUNTO 4 & $-0,166399$ & $-78,344105$ & 1475,586534 \\
\hline PUNTO 5 & $-0,1602$ & $-78,34714$ & 1297,133889 \\
\hline PUNTO 6 & $-0,154145$ & $-78,349547$ & 2925,271244 \\
\hline PUNTO 7 & $-0,146012$ & $-78,354557$ & 4278,650215 \\
\hline PUNTO 9 & $-0,135628$ & $-78,358337$ & 6725,939375 \\
\hline PUNTO 10 & $-0,131543$ & $-78,360528$ & 2053,92826 \\
\hline PUNTO 11 & $-0,125136$ & $-78,364928$ & 1616,853769 \\
\hline
\end{tabular}

DUPLICADO 2

\begin{tabular}{|l|r|r|r|}
\hline & \multicolumn{1}{|l|}{ LATITUD } & \multicolumn{1}{|l|}{ LONGITUD } & CONCENTRACION2 \\
\hline PUNTO 1 & $-0,190438$ & $-78,338554$ & 947,5507471 \\
\hline PUNTO 3 & $-0,171984$ & $-78,343045$ & 1710,602588 \\
\hline PUNTO 4 & $-0,166399$ & $-78,344105$ & 1751,172007 \\
\hline PUNTO 5 & $-0,1602$ & $-78,34714$ & 2184,318906 \\
\hline PUNTO 6 & $-0,154145$ & $-78,349547$ & 3050,676837 \\
\hline PUNTO 7 & $-0,146012$ & $-78,354557$ & 4269,332323 \\
\hline PUNTO 9 & $-0,135628$ & $-78,358337$ & 6678,755639 \\
\hline PUNTO 10 & $-0,131543$ & $-78,360528$ & 2058,346925 \\
\hline PUNTO 11 & $-0,125136$ & $-78,364928$ & 1803,328049 \\
\hline
\end{tabular}

\section{DUPLICADO 3}

\begin{tabular}{|l|r|r|r|}
\hline & LATITUD & LONGITUD & CONCENTRACION3 \\
\hline PUNTO 1 & $-0,190438$ & $-78,338554$ & 929,2068986 \\
\hline PUNTO 3 & $-0,171984$ & $-78,343045$ & 1624,292277 \\
\hline PUNTO 4 & $-0,166399$ & $-78,344105$ & 1642,403308 \\
\hline PUNTO 5 & $-0,1602$ & $-78,34714$ & 2017,815286 \\
\hline PUNTO 6 & $-0,154145$ & $-78,349547$ & 3149,045643 \\
\hline PUNTO 7 & $-0,146012$ & $-78,354557$ & 4296,59045 \\
\hline PUNTO 9 & $-0,135628$ & $-78,358337$ & 7487,993648 \\
\hline PUNTO 10 & $-0,131543$ & $-78,360528$ & 2041,83486 \\
\hline PUNTO 11 & $-0,125136$ & $-78,364928$ & 1676,626995 \\
\hline
\end{tabular}

BLANCO

\begin{tabular}{|l|r|r|r|}
\hline & LATITUD & LONGITUD & CONCENTRACION \\
\hline PUNTO 2 & $-0,104086$ & $-78,365639$ & 2245,580467 \\
\hline PUNTO 8 & $-0,135628$ & $-78,358337$ & 5880,145871 \\
\hline PUNTO 15 & $-0,183163$ & $-78,339623$ & 2706,168452 \\
\hline
\end{tabular}




\section{Conclusiones}

El método más eficiente en la evaluación de concentraciones de PM10 es el IDW (Interpolación lineal con la ponderación de la distancia inversa) por su proximidad a la concentración real de este parámetro y en cuanto a la visualización didáctica que presenta en los mapas generados en ArcGIS, permitiendo evidenciar con mayor facilidad zonas de mayor y menor concentración de PM10.

Uno de los factores por los que las concentraciones de PM10 pueden variar de un tiempo a otro es por la climatología de la zona, ya que en los meses de verano, debido a que la humedad baja, las precipitaciones son menores, la velocidad de los vientos son mayores, las partículas están suspendidas en las vías y terrenos lo que hace que la concentración tienda a subir, lo que no ocurre en los meses de invierno en los que se observa concentraciones menores de material particulado debido a que las precipitaciones permiten evitar la re suspensión de PM10 en el ambiente.

Al analizar la ubicación de estos puntos encontramos que el punto 9 uno de los de mayor concentración se encuentra en la zona de despegue de los aviones del aeropuerto de Tababela, dispersando el material particulado por el punto 10 hasta el punto 11 según la dirección del viento de la zona.

Otro punto crítico en cuanto a concentración de material particulado fue el punto ubicado en el redondel de Tababela, esto se debe a la confluencia elevada de vehículos tanto pesados como livianos provenientes de Yaruquí, Panamericana Norte y Tababela y por tanto habrá mayor emisión de PM10 por la cantidad diaria de vehículos que por el lugar circulan.

El valor de concentración en los filtros se ve influenciada por los lugares en los cuales se colocó los muestreadores caseros, enterrándolos a $1 \mathrm{~m}$ de altura sobre suelo, por lo que las partículas de polvo suspendidas y la erosión de las vías alteraron las concentraciones de PM10.

Respecto a la Norma de Calidad del Aire, publicada como parte del libro VI de Calidad Ambiental del TULSMA, se concluye que la zona del aeropuerto de Tababela supera el límite máximo permisible de $100 \mathrm{ug} / \mathrm{m}^{3}$ respecto a la norma, y en cuanto el índice de calidad de aire de la REMMAQ se considera a esta zona en emergencia debido a su posible efecto nocivo a la salud.

La Normativa ambiental vigente en nuestro país es muy permisiva, al establecer una concentración de $100 \mathrm{ug} /$ $\mathrm{m}^{3}$ como límite máximo permisible para fuentes fijas, en comparación a lo que estable la OMS de $50 \mathrm{ug} / \mathrm{m}^{3}$ de PM10 considerando a esta como causante de posibles afecciones en la salud.

El Informe Anual de Calidad del Aire en Quito establece los riesgos que se tiene con la concentración de PM10 obtenidos, las personas que se encuentren expuestas pueden contraer enfermedades respiratorias como asma o bronquitis crónica incrementando la susceptibilidad a individuos que ya tengan cuadros clínicos, agravando los mismos, con lo que se requeriría de mayores cuidados médicos e incluso se podría provocar una muerte prematura

\section{Literatura citada}

Arciniégas, C. A. 2012. Diagnóstico y control de material particulado: Luna Azul 2474(34), 2,3.

Canales-Rodríguez, M.; Quintero-Núñez, M.; CastroRomero, T. y García-Cuento, R. 2014. Las Partículas Respirables PM10 y su Composición Química en la Zona Urbana y Rural de Mexicali, Baja California en México. Información Tecnológica Vol. 25(6), 13-22. doi: 10.4067/S0718-07642014000600003

Cortés, F. \& Osses, B. 2004. Efectos de la contaminación atmosférica por material particulado en las enfermedades respiratorias agudas en menores de 5 años. Ciencia $y$ enfermeria, 10(2), 21-29.

Diaz, V. 2015. Calidad del aire en quito,Informe anual. Quito.

ESRI. 2016. ArcGIS for Desktop. Recuperado de: ArcMap: http://desktop.arcgis.com/es/arcmap/10.4/analyze/ commonly-used-tools/statistical-analysis.htm

García, F.F. 2002. Determinación de la Concentración de Fondo y Distribución Espacial de PST en Santa Marta. Grupo de Control de la Contaminación Ambiental. Universidad del Magdalena, Colombia.

Gaviria, C.F.; Benavides, P. C. \& Tangarife, C. A. 2011. Contaminación por material particulado (pm2,5 y pm10) y consultas por enfermedades respiratorias en Medellín (2008-2009). Revista Facultad Nacional de Salud Pública 29,(3), 241-250.

Golato, M.; Morales, W.; Méndez, H.; Feijóo, E. \& Paz, D. 2012. Monitoreo de emisiones de material particulado de chimeneas de generadores de vapor de la industria azucarera en Tucumán, R. Argentina. Revista industrial y agrícola de Tucumán, 83(1), 11-19.

IQCA. 2015. Indice Quiteño de la Calidad del Aire. Quito.

Maldonado, M. J. 2012. Caracterización del material particulado suspendido PM10 de la red de monitoreo de aire de la ciudad de Quito de los años 2009 y 2010 por Espectroscopía de Absorción Atómica. Quito.

Organización Mundial de la Salud [OMS]. 2005. Guías de calidad del aire de la OMS relativas al material particulado, el ozono, el dióxido de nitrógeno y el dióxido de azufre.

Ruiz, C. 2006. Caracterización del material particulado en las principales vías del transporte público colectivo y masivo del centro de Bogotá. Bogotá: Universidad de los Andes.

Secretaria de Ambiente. 2015. Informe Anual 2015 de la Calidad del Aire en Quito. Quito: Secretaria de Ambiente.

Seinfeld, J. P. 2006. Atmospheric chemistry and physics: From air pollution to climate change. John Wiley \& Sons, Inc, Hoboken. 
The Ecological Council. 2012. Contaminación del aire en los aereopuertos. Dinamarca: Lars Brogaard, 3F.

Urbaneja, L. 2016. Estimación de emisiones de los ciclos de aterrizaje y despegues de aeronaves en el aereopuerto Adolfo Suaréz Madrid- Barajas. Madrid: INDUSTRIALES-UPM.

Vivar, E. 2014. Cuantificación de material particulado pm10 y su efecto toxicológico- ambiental en la ciudad de Azogues. Azogues: Universidad de Cuenca. 